Research Article

\title{
Weak Fault Feature Extraction of Rolling Bearing Based on SVMD and Improved MOMEDA
}

\author{
Xinyu Wang (iD) and Jie Ma (iD) \\ Mechanical Electrical Engineering School, Beijing Information Science \& Technology University, Beijing 100192, China \\ Correspondence should be addressed to Jie Ma; mjbeijing@163.com
}

Received 26 June 2021; Accepted 21 October 2021; Published 28 December 2021

Academic Editor: Yong Chen

Copyright (c) 2021 Xinyu Wang and Jie Ma. This is an open access article distributed under the Creative Commons Attribution License, which permits unrestricted use, distribution, and reproduction in any medium, provided the original work is properly cited.

\begin{abstract}
In order to solve the problem that it is very difficult to extract fault features directly from the weak impact component of early fault signal of rolling bearing, a method combining continuous variational mode decomposition (SVMD) with modified MOMEDA based on Teager energy operator is proposed. Firstly, the low resonance impulse component in the fault signal is separated from the harmonic component and noise by SVMD, and then the Teager energy operator is used to enhance the impulse feature in the low resonance component to ensure that the accurate fault period is selected by the MOMOEDA algorithm. After further noise reduction by MOMEDA, the envelope spectrum of the signal is analyzed, and finally the fault location is determined. The results of simulation and experimental data show that this method can accurately and effectively extract the characteristic frequency of rolling bearing weak fault.
\end{abstract}

\section{Introduction}

Rolling bearing is one of the important parts of rotating machinery, which ensures the working accuracy of the shaft [1]. When the bearing is partially damaged, the vibration signal will produce periodic impact characteristics, which will affect the normal operation of the equipment. Therefore, accurate and timely identification of bearing faults can effectively ensure the safety of equipment operation. However, early fault vibration signals often have nonlinear and nonstationary characteristics, the impact component of fault characteristics is easy to be submerged in strong background noise, and it is difficult to judge the fault type directly from time domain or frequency domain $[2,3]$. Therefore, the early fault diagnosis of rolling bearing has always been a research difficulty and hot spot.

Empirical mode decomposition (EMD) can express nonlinear and nonstationary signals as the sum of physically averaged time-frequency components, which has been applied satisfactorily in many applications [4]. However, the results of EMD are highly influenced by the searching of extremum and interpolation methods. Different from EMD, variational mode decomposition (VMD) is a nonrecursive decomposition method, which compresses different frequency bands around different center frequencies [5]. However, both VMD and its improved algorithm are based on the assumption that the modal component is narrow band. In reference [6], an extension of VMD is given to decompose the signal containing broadband nonlinear frequency modulation component, that is, variational nonlinear frequency modulation mode decomposition (VNCMD). It is a method to transform the wideband Nonlinear FM component into narrow band signal for analysis by demodulation technology [6]. Recently, a new method, variational mode extraction (VME), is proposed to extract the intrinsic mode function by knowing the approximate central frequency of the intrinsic mode function [7]. Later, continuous variational mode decomposition (SVMD) algorithm is extended to VME, which is an efficient and fast adaptive signal variational decomposition method. This new decomposition method extracts all the modal components (IMF) in a continuous way, does not need to know the number of modes, and has low computational complexity [8]. 
In addition, the transmission process of the original fault signal of rolling bearing can be regarded as a linear convolution mixing process of the original signal and the channel, and the extraction of the original fault impact signal can be regarded as a deconvolution process. From this point of view, Wiggins [9] proposed minimum entropy deconvolution (MED) algorithm for the first time, which uses kurtosis maximization to recover the pulse like estimation of the original vibration signal. However, the results of MED cannot reflect the real situation of bearing failure. Later, Wiggins [10] and others improved MED and proposed a maximum correlation kurtosis deconvolution algorithm (MCKD). Based on correlation kurtosis, we can measure the impulsivity of signals correlated with a given period, but improper selection of parameters will lead to poor estimation of correlation kurtosis. An optimal minimum entropy adjusted deconvolution algorithm (MOMEDA) is proposed by Cabrelli [11], which proves that the output returned by OMEDA has a simpler structure. However, OMEDA only tends to deconvolute a single pulse effectively. Therefore, McDonald and Zhao [12] proposed an improved algorithm of OMEDA, which overcomes the limitations of MED, MCKD, and OMEDA by introducing the target vector to deconvolute the ideal periodic pulse sequence. However, when the early fault signal is directly denoised by MOMEDA algorithm, it is easy to be interfered by strong background noise.

Based on the above analysis, using Teager energy operator has strong antinoise ability and can enhance the property of transient impact [13-15]; using MOMEDA to highlight fault impact features from Teager energy operator can extract weak fault frequency more effectively. The SVMD algorithm is combined with the improved MOMEDA algorithm, and the simulation results are compared with other diagnosis methods to verify the effectiveness and practicability of the proposed method.

\section{Fundamental Theory}

2.1. SVMD. SVMD algorithm can be regarded as a continuous implementation of VMD and an extension of VME. In this method, some criteria are added to the VMD algorithm to ensure that the latest modal components are different from those previously found. The biggest advantage of SVMD algorithm is that it does not need to know the number of available modal components in the signal, but it is a key parameter for VMD algorithm.

In this algorithm, VME is applied to decompose the signal in turn, and some constraints are added to avoid converging to the previously extracted modal components. This process will continue until all modal components are extracted or the reconstruction error (the error between the sum of the input signal and the modulus) is less than the threshold. Mathematically, it is assumed that the input signal $f(t)$ is decomposed into two signals: the $L$ th modal component $u_{L}(t)$ and the residual signal $f_{r}(t)$, as follows:

$$
f(t)=u_{L}(t)+f_{r}(t)
$$

where $f_{r}(t)$ is an input signal other than $u_{L}(t)$ and contains two parts: the sum of the previously obtained moduli and the unprocessed part of the signal, namely,

$$
f_{r}(t)=\sum_{i=1: L-1} u_{i}(t)+f_{u}(t)
$$

Obviously, in order to find the first modal component, the first part of $f_{r}(t)$ (the sum of the modal components obtained previously) is zero. An iteration of SVMD can be completed by the following equation:

$$
\widehat{u}_{L}^{n+1}(\omega)=\frac{\hat{f}(\omega)+\alpha^{2}\left(\omega-\omega_{L}^{n}\right)^{4} \hat{u}_{L}^{n}(\omega)+\hat{\lambda}(\omega) / 2}{\left[1+\alpha^{2}\left(\omega-\omega_{L}^{n}\right)^{4}\right]\left[1+2 \alpha\left(\omega-\omega_{L}^{n}\right)^{2}+\sum_{i=1}^{L-1} 1 / \alpha^{2}\left(\omega-\omega_{i}\right)^{4}\right]}
$$

where $\alpha$ denotes the equilibrium parameters of data fidelity constraints, which can be solved by the Lagrange multiplier method. As explained in [5], $\alpha$ the value of $\alpha$ is usually very large. Therefore, the equation of updating $\omega$. can be approximately expressed as

$$
\omega_{L}^{n+1}=\frac{\int_{0}^{\infty} \omega\left|\hat{u}_{L}^{n+1}(\omega)\right|^{2} \mathrm{~d} \omega}{\int_{0}^{\infty}\left|\hat{u}_{L}^{n+1}(\omega)\right|^{2} \mathrm{~d} \omega}
$$

Finally, Lagrange multiplier is given $\lambda$. The modified equation of the method is obtained by the double rising method

$$
\hat{\lambda}^{n+1}=\hat{\lambda}^{n}+\tau\left[\hat{f}(\omega)-\left(\hat{u}_{L}^{n+1}(\omega)+f_{u}^{n+1}(t)+\sum_{i=1}^{L-1} u_{i}^{n+1}(\omega)\right)\right],
$$

where $\tau$ represents the update parameter. 


$$
\left\{\begin{array}{l}
y(\omega)=\frac{\alpha^{2}\left(\omega-\omega_{L}^{n+1}\right)^{4}\left(\hat{f}(\omega)-\widehat{u}_{L}^{n+1}(\omega)-\sum_{i=1}^{L-1} \widehat{u}_{i}(\omega)+\widehat{\lambda}(\omega) / 2\right)-\sum_{i=1}^{L-1} \widehat{u}_{i}(\omega)}{1+\alpha^{2}\left(\omega-\omega_{L}^{n+1}\right)^{4}}, \\
\hat{\lambda}^{n+1}=\hat{\lambda}^{n}+\tau\left[\hat{f}(\omega)-\left(\widehat{u}_{L}^{n+1}(\omega)+y(\omega)+\sum_{i=1}^{L-1} u_{i}^{n+1}(\omega)\right)\right] .
\end{array}\right.
$$

The SVMD algorithm extracts modal components one by one until the reconstruction error reaches a certain threshold. The optimization problem in SVMD can be approximately regarded as a k-dimensional optimization problem at each frequency, while VMD is a k-dimensional optimization problem [8], which further shows that SVMD has lower computational complexity.

2.2. Teager Energy Operator. Teager energy operator is a kind of nonlinear differential operator, which can amplify the transient energy component of the signal by nonlinear combination of the instantaneous value and its differential of the vibration signal, which can more highlight the instantaneous characteristics of the impact [13].

For continuous time signals $x(t)=a(t) \cos [\Phi(t)]$, Teager energy operator is defined as

$$
\psi[x(t)]=\left[\dot{x}(t)^{2}\right]-x(t) \ddot{x}(t),
$$

where $\dot{x}(t)$ and $\ddot{x}(t)$ are the first and second derivatives of signal $x(t)$.

If $x(t)$ is a discrete signal, the differential is replaced by difference, and the Teager energy operator is approximately defined as

$$
\psi[x(n)]=[x(n)]^{2}-x(n+1) x(n-1) .
$$

It can be seen from equation (8) that for discrete-time signals, the Teager energy at the current time can be calculated as long as the samples at the current time and before and after the time are known.

The output of Teager energy operator is the product of the instantaneous amplitude and the square of the instantaneous frequency, which increases the product of the square of the frequency compared with the traditional energy definition [14]. Since the vibration frequency of transient shock is high, the output of Teager energy operator can effectively enhance the transient shock component.

Aiming at the problem of rolling bearing fault feature extraction under strong background noise, the instantaneous Teager energy operator can be used, which has the advantages of good time resolution and adaptive ability to the transient change of signal, highlighting the impact fault feature of bearing [14].

2.3. MOMEDA. The essence of MOMEDA is to recover the impulse characteristics of the original signal by finding an optimal filter $f$ in a noniterative way, so as to minimize the impact of noise on the extracted impulse signal, which is a deconvolution process [16]. The process of shock signal transmission from signal source to sensor can be expressed as follows:

$$
x=h * y+e,
$$

where $y$ expresses the bearing fault impact signal; $h$ expresses the system transfer function; $x$ expresses the original vibration signal collected by sensor; and $e$ expresses the random noise.

The deconvolution process is as follows:

$$
y=f * x=\sum_{k=1}^{N-L} f_{k} x_{k+L-1},
$$

where $k$ expresses the total sampling points, $k=1,2, \ldots, n-1$, and $L$ expresses the filter length.

For the characteristics of periodic pulse signals of rotating machinery, the method of MOMEDA defines multipoint D-norm based on D-norm:

$$
\begin{aligned}
\operatorname{MDN}(y, t) & =\frac{1}{\|t\|} \frac{t^{T} y}{\|y\|}, \\
\operatorname{MOMEDA}(y, t) & =\max _{f} \operatorname{MDN}(y, t)=\frac{1}{\|t\|} \frac{t^{T} y}{\|y\|},
\end{aligned}
$$

where $y$ expresses the vibration signal vector; $f$ expresses the filter vector bank; and $t$ expresses the target vector, used to determine the pulse position and weight of deconvolution target.

When the fault impulse signal is completely coincident with the target vector $t$, the multipoint $\mathrm{D}$-norm reaches the maximum, the corresponding filter $f$ is the optimal filter, and the deconvolution effect is optimal.

The extremum of equation (12) is obtained by deriving the filter:

$$
\begin{aligned}
\frac{d}{\mathrm{~d} f}\left(\frac{t^{T} y}{\|y\|}\right)= & \|y\|^{-1}\left(t_{1} M_{1}+t_{2} M_{2}+\cdots+t_{k} M_{k}\right) \\
& -\|y\|^{-3} t^{T} y X_{0} y=0,
\end{aligned}
$$

where $f=f_{1}, f_{2}, \ldots, f_{L} ; t=t_{1}, t_{2}, \ldots, t_{N-L} ; M_{k}=\left[\begin{array}{c}x_{k+L-1} \\ x_{k+L-2} \\ \vdots \\ x_{k}\end{array}\right]$. 

that

By further simplifying equation (13), it can be concluded

$$
\frac{t y}{\|y\|^{2}} X_{0} y=X_{0} t
$$

where $X_{0}$ is the matrix form of pulse signal, $X_{0} t=t_{1} M_{1}+t_{2} M_{2}++t_{k} M_{k_{\circ}}$.

Suppose that the inverse toplitz autocorrelation matrix $(X 0 X T 0)^{-1}$ exists, and $y=X T$ of is substituted into equation (14):

$$
\frac{t y}{\|y\|^{2}} f=\left(X_{0} X_{0}^{T}\right)^{-1} X_{0} t
$$

From the above formula, we can take its special solution $f$ as a group of optimal filters, which is the solution of MOMEDA:

$$
f=\left(X_{0} X_{0}^{T}\right)^{-1} X_{0} t
$$

By substituting equation (16) into $y=X T$ Tf, the original shock signal can be recovered to the greatest extent.

\subsection{Early Fault Feature Extraction of Rolling Bearing. The} impact component of rolling bearing early signal is weak, so it is more difficult to extract fault features under strong background noise. Because the VMD method decomposes each modal component (IMF) at the same time, when the number of available modulus in the signal is high, the calculation time will increase significantly, and some of the decomposed modal components are regarded as interference or noise signals. SVMD is an algorithm for searching modal components step by step. This continuity helps to improve the convergence speed. At the same time, it does not extract unnecessary modal components and reduces the calculation time. Therefore, SVMD algorithm is better than VMD algorithm.

In addition, although MOMEDA algorithm has many advantages, it is easy to be interfered by strong background noise when extracting fault period directly from multipoint kurtosis spectrum of early fault signal, which leads to extracting noise period instead of fault period. Therefore, the Teager energy operator of the original signal is used to enhance the periodic fault impulse component in the signal, and then the fault period is extracted from the multipoint kurtosis spectrum of the Teager energy operator by using MOMEDA, which can achieve efficient noise reduction. The specific process is as follows:

(1) Reasonable parameters such as balance parameter, time step of double lifting, tolerance of convergence criterion, and type of stop criterion are set for SVMD algorithm, and a series of IMF components are obtained by decomposing fault signal with SVMD

(2) The sum of all components is used to reconstruct, and the Hilbert envelope demodulation analysis is used to determine whether the fault characteristic frequency can be extracted
(3) If the fault characteristic frequency cannot be extracted, the Teager energy operator of the reconstructed signal is calculated to highlight the continuous periodic impact component in the reconstructed fault signal

(4) Combined with the theoretical fault period, the reasonable period interval and filter length are set, and the noise reduction and feature enhancement of Teager energy operator are further carried out by using MOMEDA algorithm

(5) The Hilbert envelope is used to demodulate the above filtering signal, and the fault location is diagnosed by comparing with the theoretical fault characteristic frequency

The detailed fault diagnosis flow of the proposed method is shown in Figure 1.

Here, error rate $\eta$ of the theoretical failure period $\delta$ and the actual failure cycle $\omega$ is introduced, as an index to measure the deviation between the actual cycle and the theoretical cycle. Error rate $\eta$ is defined as

$$
\eta=\frac{|\delta-\omega|}{\delta} \times 100 \%
$$

\section{Simulation Verification}

In order to verify the noise reduction effect based on the combination of SVMD and improved MOMEDA algorithm, the following simulation signals are used to simulate the weak fault of rolling bearing:

$$
\left\{\begin{array}{l}
x(t)=s_{0} e^{-2 \pi \zeta f_{n} t} \sin \left(2 \pi f_{n} \sqrt{1-\zeta^{2}} t\right), \\
y(t)=x(t)+n(t),
\end{array}\right.
$$

where $s_{0}$ expresses the displacement constant, $s_{0}=2 ; \zeta$ expresses the damping coefficient, $\zeta=0.1$; FN expresses the natural frequency of bearing, $f_{n}=2000 \mathrm{~Hz} ; x(t)$ expresses the periodic shock component; $n(t)$ expresses the Gaussian white noise; the signal-to-noise ratio of the simulation signal is $-13 \mathrm{~dB}$; the known fault characteristic frequency $f=100 \mathrm{~Hz}$; and the number of sampling points $N=5120$.

The time-domain waveform of the signal shown in Figure 2 is obtained by simulation. Due to the heavy white Gaussian noise added to the impact signal, the original impact component has been completely submerged in the noise signal, and it is difficult to find obvious periodic impact characteristics.

It can be seen from the spectrum and envelope spectrum of the simulation signal in Figure 3 that the characteristic frequency and its frequency doubling of the impulse signal are affected by the frequency conversion modulation phenomenon and strong background noise, and the obvious fault characteristic frequency and its frequency doubling component cannot be found in the envelope spectrum.

From the above analysis, it can be seen that it is difficult to identify the rolling bearing fault simply by using envelope spectrum, so it is necessary to denoise the fault signal before 


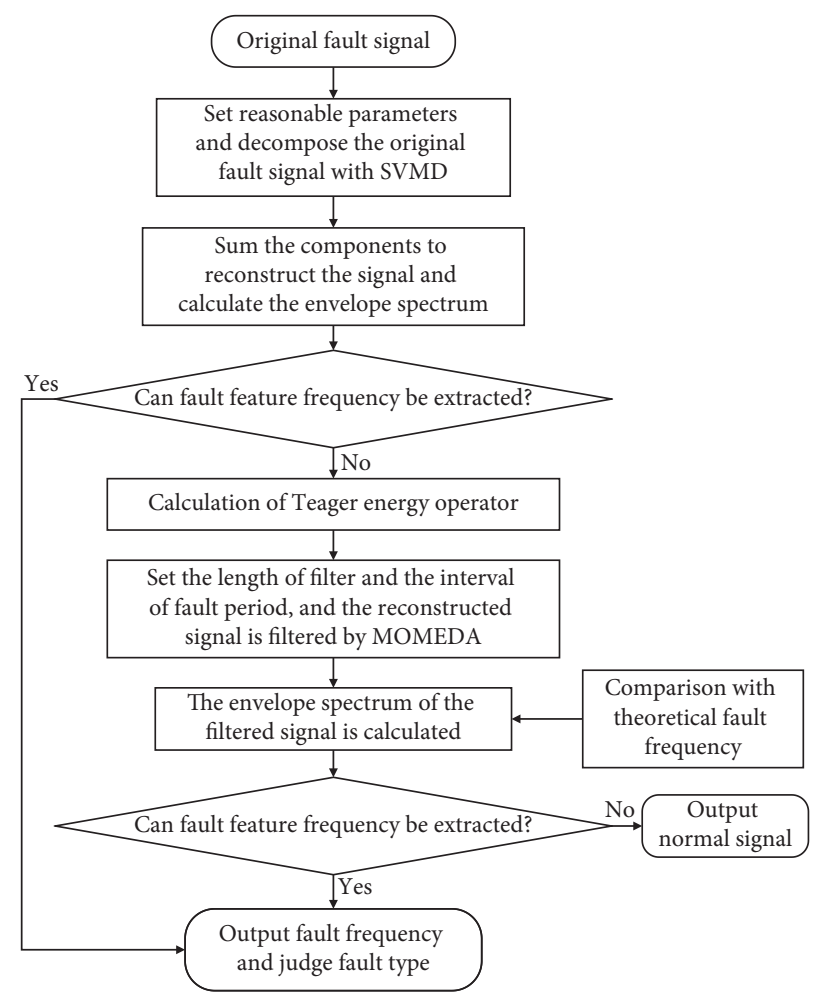

FIGURE 1: Fault diagnosis flow chart.

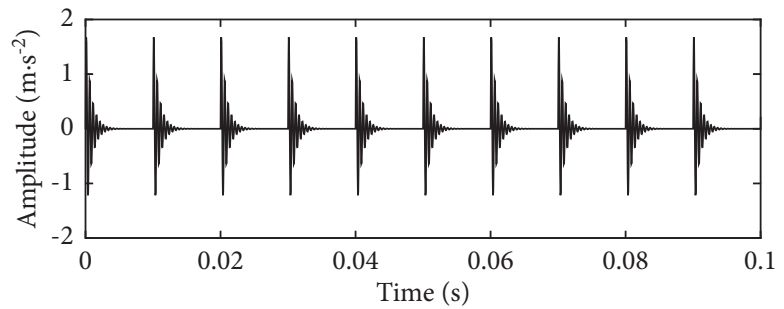

(a)

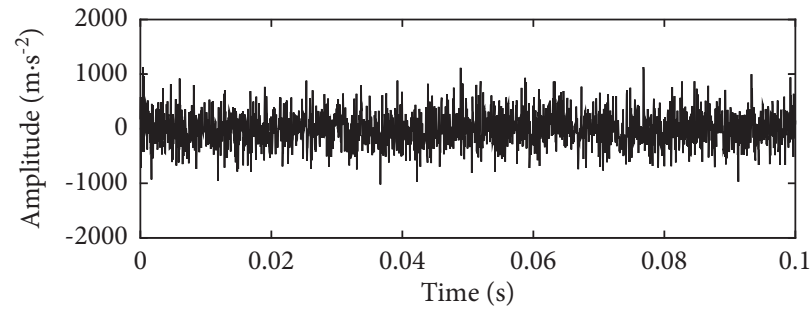

(b)

Figure 2: Time-domain waveform of simulation signal. (a) Signal without noise. (b) Signal with strong noise.

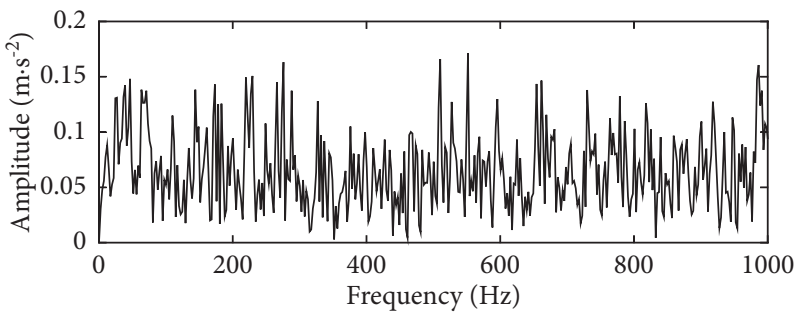

(a)

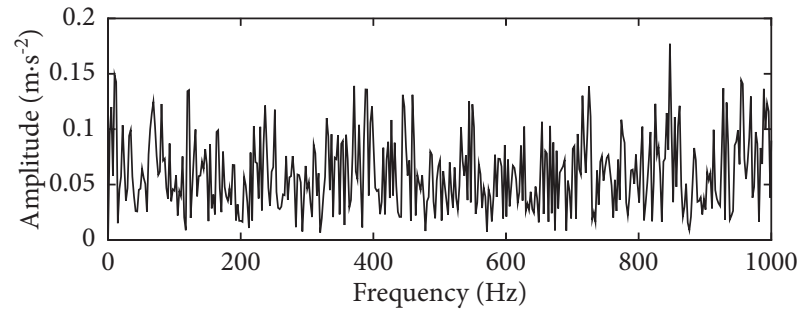

(b)

Figure 3: Frequency domain waveform of simulated signal. (a) Spectrum. (b) Envelope spectrum.

envelope analysis, so as to improve the signal-to-noise ratio and enhance the fault impact component. The simulation signal is decomposed by SVMD to separate weak fault features and noise interference frequency. First, set the maximum balance parameters $\alpha=2000$. The obtained components (IMF1-IMF5) are shown in Figure 4. Since all the components extracted by SVMD are needed, the reconstructed signal and its envelope spectrum can be obtained by summation of each component signal directly, as shown in Figure 5.

The fault characteristic frequency is shown in Figure 5, which shows that SVMD decomposition has a certain effect. 


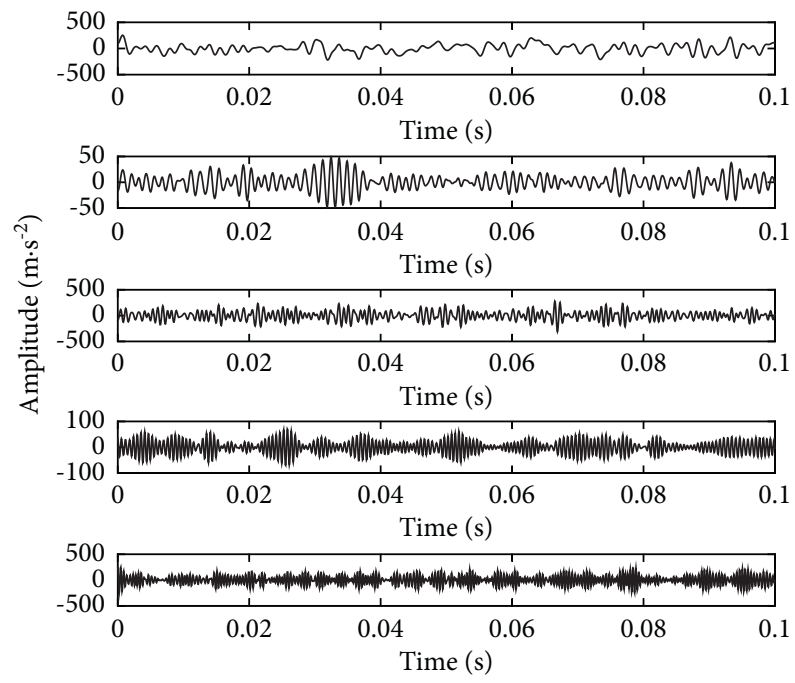

FIgURE 4: Decomposition process of SVMD.

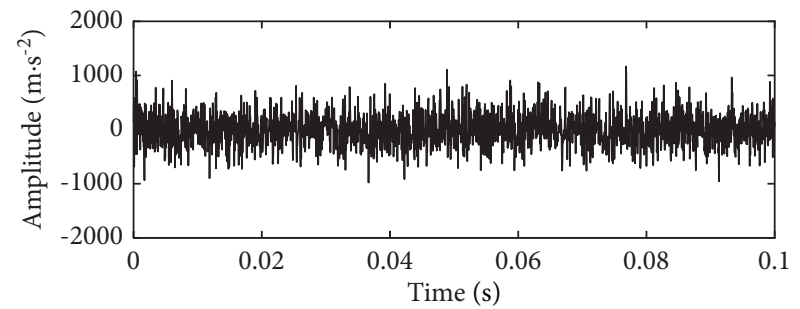

(a)

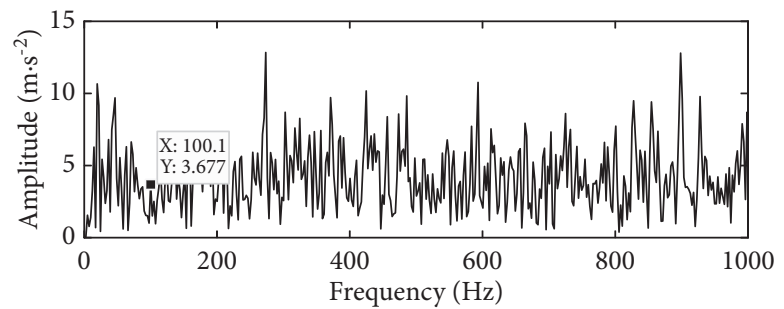

(b)

FIGURE 5: Reconstructed signal. (a) Time-domain waveform. (b) Envelope spectrum.

However, the amplitude of the interference frequency around the fault feature of the signal is also large, and its frequency doubling component cannot be found accurately, so it is necessary to further denoise the fault signal after decomposition and reconstruction.

The multipoint kurtosis spectrum and interval diagram of Teager energy operator of reconstructed signal are shown in Figure 6. It can be seen from the figure that the points with large multipoint kurtosis value can be observed in the multipoint kurtosis spectrum of Teager energy operator, and the corresponding periods are $T_{1}=101, T_{2}=201$, and $T_{3}=301$, respectively, corresponding to $0.5,1$, and 1.5 times of the theoretical fault period. The period corresponding to the maximum kurtosis value $\left(T_{3}=201\right)$ can be observed clearly in the period interval $[150,250]$, and the periodic error rate at this time $\eta=5 \%$, which is basically consistent with the theoretical failure period, that is, $T_{2}=201$ as the accurate failure period.

The time-domain waveform of Teager energy operator is extracted by using MOMEDA algorithm and its envelope spectrum is calculated. Set the filter length of MOMEDA to 1000 and the fault period interval to $[195,205]$. The results are shown in Figure 7. In Figure 7(b), a number of impact components with regular attenuation can be observed, corresponding to the frequency doubling $f \sim 7 f$ of the fault characteristic frequency of the simulation signal, and the fault type can be judged according to this.
Compared with Figures 5 and 7, it is found that the periodic pulse component of the time-domain waveform obtained by the proposed method is more obvious, and the fault characteristic frequency and its frequency doubling component of the envelope spectrum have also been accurately extracted. In order to further verify the effectiveness of the proposed method, it is compared with the method based on SVMD and MOMEDA. The noise reduction signal and its envelope spectrum are obtained by MOMEDA filtering on the reconstructed SVMD signal (the parameter settings are consistent), as shown in Figure 8.

Compared with Figures 7 and 8 , it is found that the method of SVMD and improved MOMEDA is obviously better than the comparison method. Firstly, the periodic impulse characteristics of the signal obtained by the proposed method are more prominent in the time-domain diagram, and the peak values of the fault characteristic frequency and its frequency doubling components extracted from the envelope spectrum are more obvious, which further verifies the effectiveness of the proposed method.

\section{Experimental Verification}

The method proposed in this paper is verified by using the bearing open data set of Case Western Reserve University and 


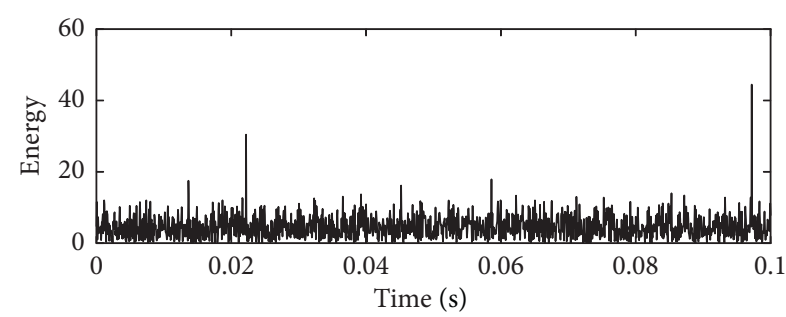

(a)

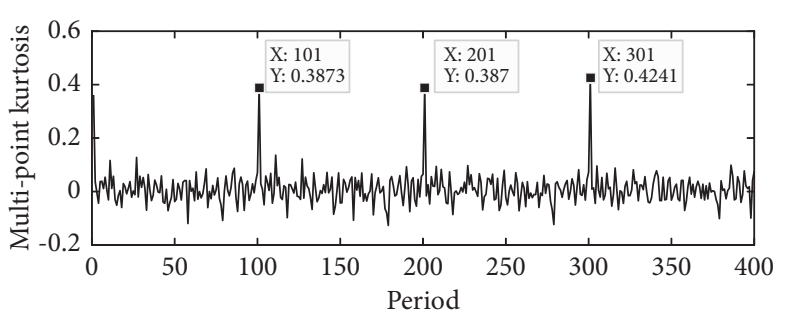

(b)

Figure 6: Teager energy operator and its multipoint kurtosis spectrum of reconstructed signal. (a) Teager energy operator. (b) Multipoint kurtosis spectrum.

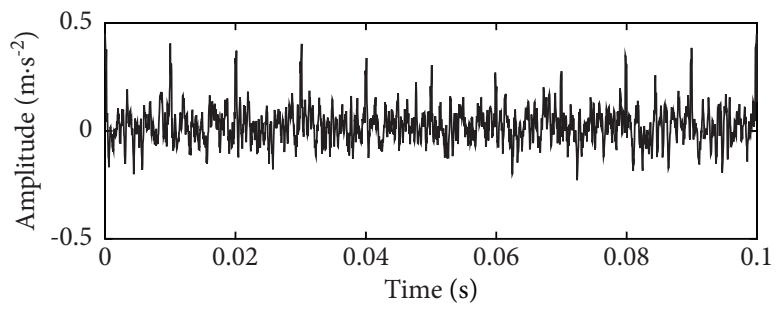

(a)

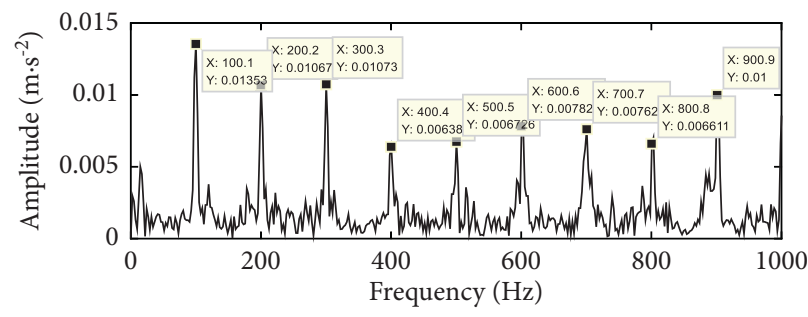

(b)

FIgure 7: Signal based on SVMD and improved MOMEDA. (a) Time-domain waveform. (b) Envelope spectrum.

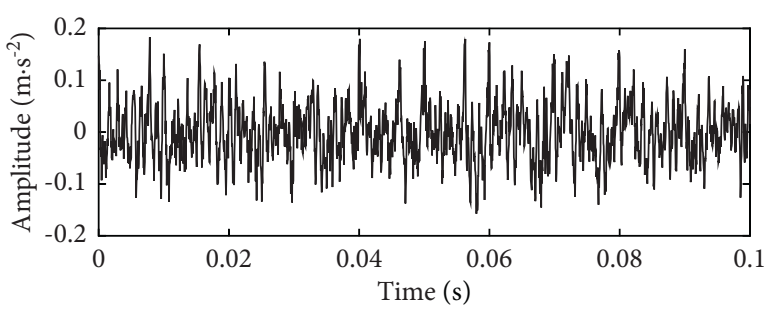

(a)

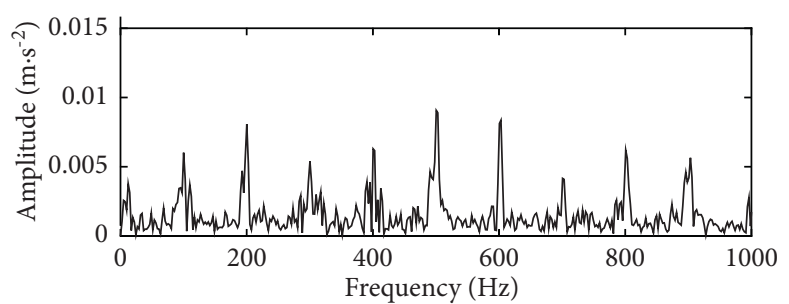

(b)

Figure 8: Signal based on SVMD and MOMEDA.

(a) Time-domain waveform. (b) Envelope spectrum.

compared with other methods. In addition, the data come from the official website of bearing data of Western Reserve University: https://csegroups.case.edu/bearingdatacenter/home.

4.1. Early Fault Data Description. In the experiment, 62032RS JEM SKF deep groove ball bearing was selected, and the specific parameters are shown in Table 1.

The motor speed is $1750 \mathrm{r} / \mathrm{min}$, the rotation frequency is $29.17 \mathrm{~Hz}$, and the sensor sampling frequency is $12 \mathrm{KHz}$. For the convenience of experimental calculation, 24000 points of inner ring fault data are selected. According to the following formula of fault frequency, the failure frequency of inner ring of rolling bearing can be calculated as $144.3 \mathrm{~Hz}$.

$$
f_{i}=0.5 \mathrm{rn}\left(1+\frac{d}{D} \cos \alpha\right),
$$

where $d$ expresses the rolling body diameter; $D$ expresses the bearing feature diameter; $\alpha$ expresses the bearing contact angle; and $n$ expresses the number of rolling bodies, and $r$ represents the transition. Early fault time-domain signals and their envelope are shown in Figure 9.
Observing Figure 9, it is difficult to observe obvious and regular periodic impact characteristics in time-domain waveform because the impact component in original fault signal is submerged by strong background noise. Moreover, only the motor rotation frequency $f r$ and its frequency doubling can be observed in the envelope spectrum, and the rolling bearing fault characteristic frequency and its frequency doubling component are difficult to be effectively extracted due to the occurrence of frequency component modulation such as noise. Therefore, it is necessary to use the proposed method to denoise the original fault signal.

4.2. Early Fault Analysis Based on SVMD. Because the envelope spectrum of the original signal cannot directly reflect the fault state information, a new signal decomposition method, SVMD decomposition, is used for preliminary noise reduction. First, set the maximum balance parameters $\alpha=12000$. The time step of double promotion $t a u=0$. The tolerance of convergence criterion tol=1e-6. The stop criterion type stopc $=4$ (converges to the energy of the last modal component), and then SVMD decomposition is 
TABLE 1: Structural parameters of 6203-2RS JEM SKF deep groove ball bearing.

\begin{tabular}{lcccc}
\hline Bearing type & Diameter of rolling element $(\mathrm{mm})$ & Bearing pitch diameter $(\mathrm{mm})$ & Bearing contact angle & Number of rolling \\
\hline SKF 6203 & 6.75 & 28.5 & $0^{\circ}$ & 8 \\
\hline
\end{tabular}

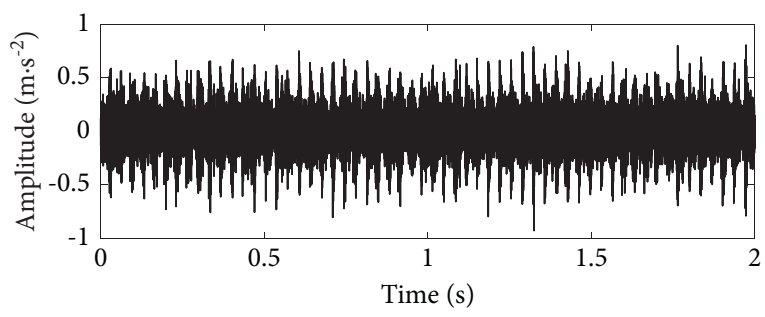

(a)

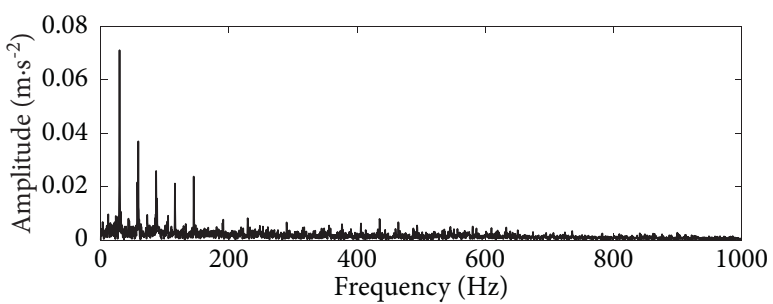

(b)

Figure 9: Early fault signal. (a) Time-domain waveform. (b) Envelope spectrum.

performed to obtain 11 modal components (IMF1-IMF11), the first five of which are shown in Figure 10. Finally, according to the theory that all components extracted by SVMD algorithm are valid, the SVMD reconstructed signal and its envelope spectrum are obtained by summing all components, as shown in Figure 11.

It can be seen from Figure 10 that the impact component of the signal decomposed and reconstructed by SVMD is more prominent in the time-domain diagram, and the fault characteristic frequency and its frequency doubling component of the signal can be preliminarily found in the envelope spectrum, which shows that the decomposition method has a certain effect on the noise reduction of the signal. However, the peak value of fault characteristic frequency and its frequency doubling is not prominent, especially the weak signal can only be found at the frequency doubling, which is easily submerged by noise. Therefore, this method cannot accurately and effectively extract the early fault characteristic frequency of rolling bearing. It is necessary to take further noise reduction methods to extract the fault characteristic frequency more accurately.

4.3. Early Fault Analysis Based on SVMD and Improved $M O M E D A$. In order to highlight the characteristic frequency and frequency doubling component of the early fault signal of rolling bearing, the improved MOMEDA method is adopted to further enhance the signal characteristics after SVMD decomposition and reconstruction. Firstly, the Teager energy operator and its multipoint kurtosis spectrum of SVMD reconstructed signal are calculated, and the results are shown in Figure 12.

It can be seen from Figure 12 that the significantly enhanced impact component can be observed in the signal Teager energy operator diagram. In the multipoint kurtosis spectrum, the peak values of multipoint kurtosis with corresponding periods of 40,84 , and 167 are $0.5,1$, and 2 times of the theoretical fault period, respectively. In the period interval $[80,86]$, it is obvious that the period corresponding to the maximum kurtosis value is 84 , and the periodic error rate is lower $\eta=1.01 \%$, which is basically consistent with the

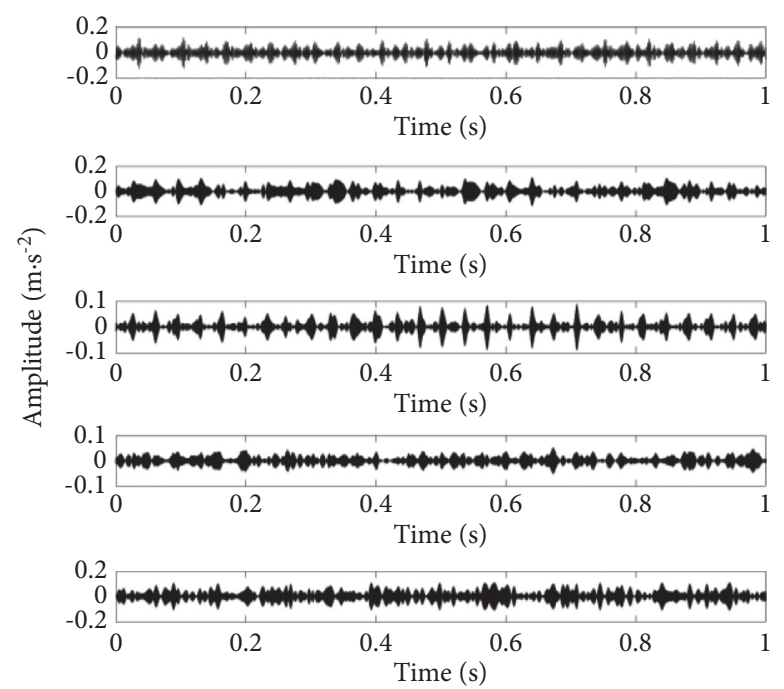

FIGURE 10: SVMD decomposition process.

theoretical failure cycle. It is judged that the selected period is the accurate failure period.

The time-domain diagram and envelope spectrum of Teager energy operator extracted by MOMEDA filtering are shown in Figure 13. Obvious periodic impact component can be observed from the time-domain diagram, and the noise interference in the envelope spectrum can be significantly reduced. Obvious impact component $(145 \mathrm{~Hz})$ can be observed, which basically corresponds to the theoretical fault frequency $144.3 \mathrm{~Hz}$ of inner ring and its multiple frequency multiplication. According to this, it can be judged that there is inner ring fault in this rolling bearing.

In order to further prove the superiority of the proposed method, it is compared with other fault feature extraction methods. The time-domain signal and envelope spectrum are obtained by using the method based on SVMD and MOMEDA, as shown in Figure 14. Compared with the proposed method, it can be seen from the envelope spectrum that this method can only extract a single frequency of bearing early fault, and its frequency doubling component is easily submerged by noise, so it cannot be effectively 


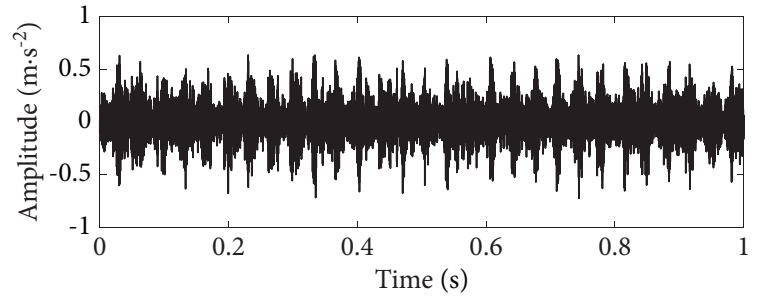

(a)

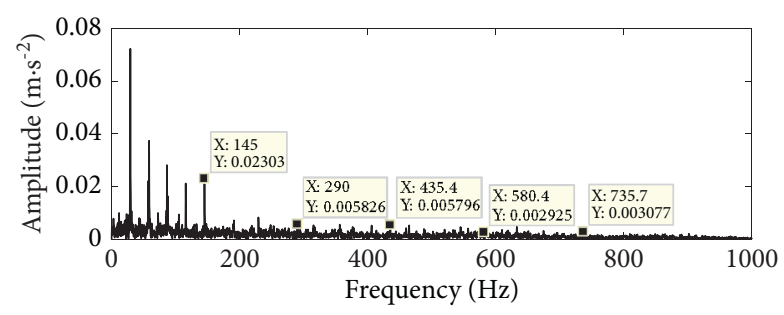

(b)

FIgURE 11: Signal reconstructed after SVMD decomposition. (a) Time-domain waveform. (b) Envelope spectrum.

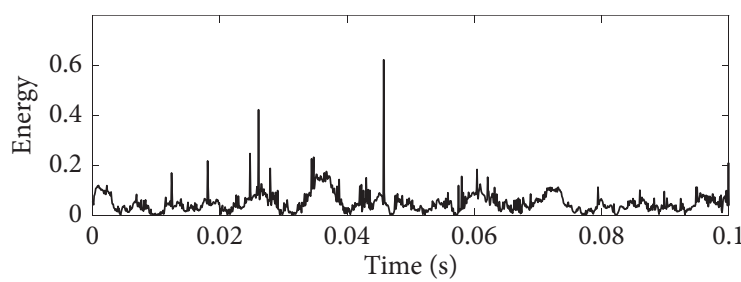

(a)

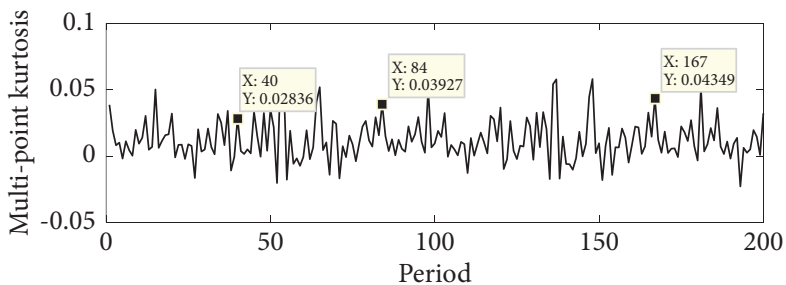

(b)

Figure 12: Teager energy operator and its multipoint kurtosis spectrum of reconstructed signal. (a) Teager energy operator. (b) Multipoint kurtosis spectrum.

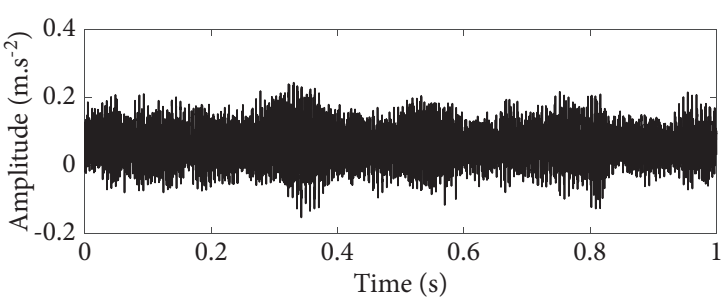

(a)

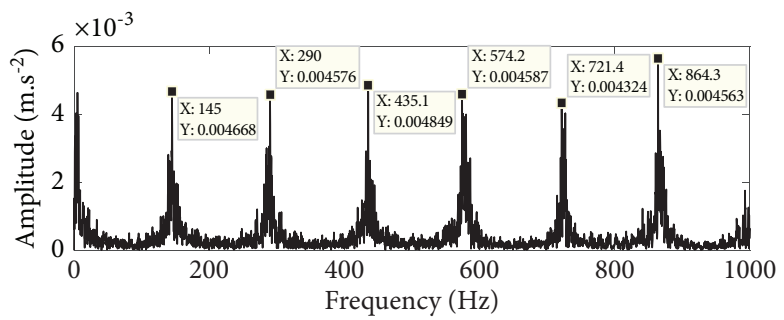

(b)

Figure 13: Signal based on SVMD and improved MOMEDA. (a) Time-domain waveform. (b) Envelope spectrum.

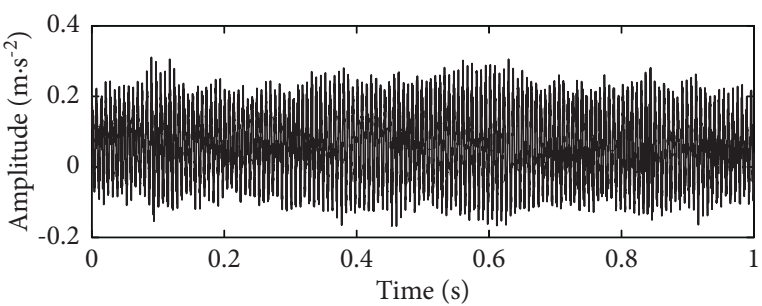

(a)

Figure 14: Signal based on SVMD and MOMEDA.

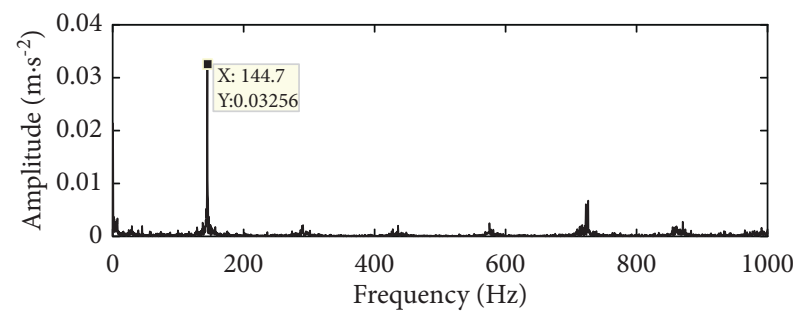

(b)

(a) Time-domain waveform. (b) Envelope spectrum.

extracted. Then, the method based on SVMD and MCKD is used to extract the characteristic frequency of the original fault signal (set the filter length as 100 and the number of iterations as 200), and the time-domain signal and envelope spectrum are obtained, as shown in Figure 15.

It can be seen from the time-domain waveform in Figure 15 that only a small number of periodic impact components appear in the deconvolution reconstruction signal of MCKD, which indicates that the deconvolution effect is not ideal. The fault characteristic frequency can be found in the envelope spectrum, but its amplitude is very small, and its frequency doubling cannot be accurately found, and the characteristic frequency is easily interfered by noise and other modulation frequencies. The effectiveness of the improved MOMEDA method is further verified.

In addition, the time-domain waveform and envelope spectrum of the signal are obtained by using the method proposed in reference [17], as shown in Figure 16. Although 


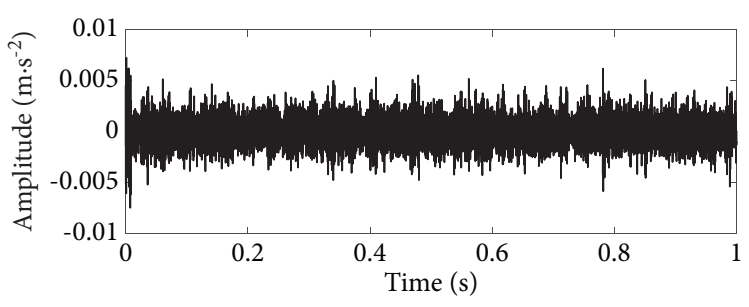

(a)

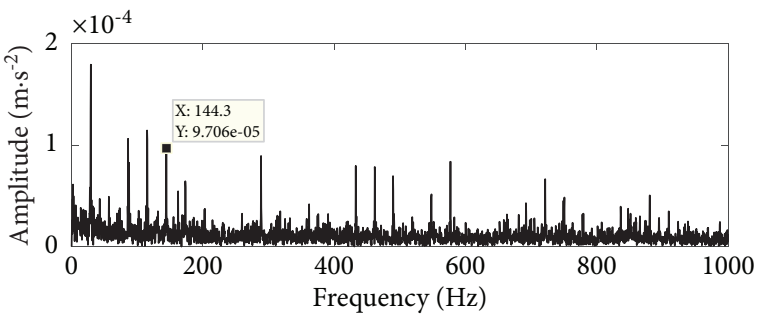

(b)

Figure 15: Signal based on SVMD and MCKD. (a) Time-domain waveform. (b) Envelope spectrum.

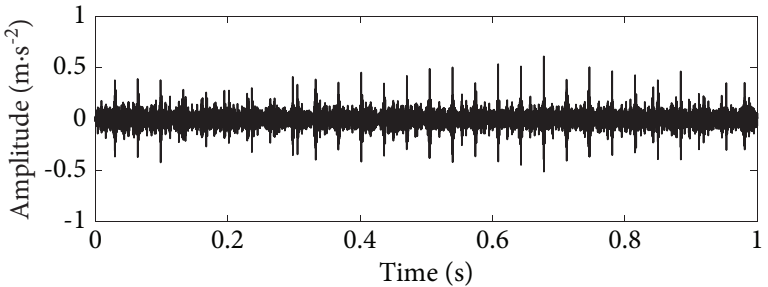

(a)

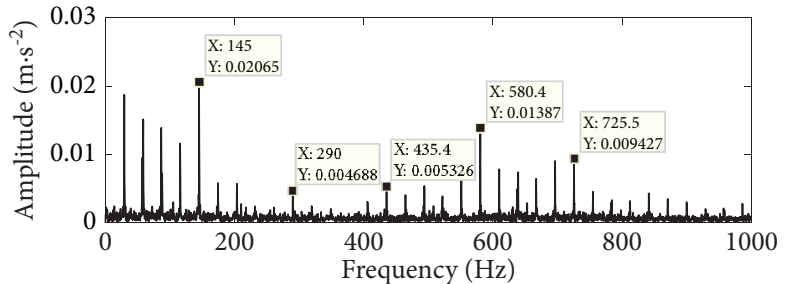

(b)

FIGURE 16: Signal based on adaptive MCKD and CEEMDAN. (a) Time-domain waveform. (b) Envelope spectrum.

the fault impulse component can be seen in the time-domain diagram, the fault characteristic frequency and its frequency doubling component obtained by the proposed method are weaker than those obtained by the proposed method in the envelope spectrum, especially the frequency doubling component is easily interfered by other frequency components, which further illustrates the effectiveness of the proposed method.

By comparing the proposed method with the other three feature extraction methods, it is found that the proposed method is the least disturbed by strong background noise, and the peak value is the most prominent at the fault feature frequency and its frequency doubling, which can accurately extract the early fault feature frequency of rolling bearing. The effectiveness and practicability of the method based on SVMD and improved MOMEDA are fully proved.

\section{Conclusion}

This paper presents a bearing fault diagnosis method based on SVMD and improved MOMEDA. Firstly, SVMD technology is used to decompose the vibration signal of the bearing, and several IMF components are obtained. Considering that the components extracted by SVMD method are all useful, the fault impulse signal is reconstructed directly by summing the components. Then, the improved MOMEDA is used to denoise the reconstructed signal. Finally, the denoised signal is demodulated by Hilbert envelope.

The simulation and experimental results show that compared with VMD, the most advantage of SVMD is that it does not need to know the number of available modes in the signal, and it has lower computational complexity and can successfully converge to the real modal components. In addition, compared with MOMEDA and other deconvolution algorithms, the improved MOMEDA algorithm can highlight the fault characteristic frequency when dealing with periodic pulse signals with strong background noise. The interference components near the peak are few, and the characteristic frequency and frequency doubling characteristics are greatly enhanced, which is more convenient for fault analysis.

Through the analysis of simulation signals and experimental data, compared with other rolling bearing fault feature extraction methods, the proposed method can extract fault feature frequency more accurately under strong noise interference, and the time cost is lower, which verifies the effectiveness and practicability of the method.

\section{Data Availability}

The data used to support the findings of this study are available from the corresponding author upon request.

\section{Conflicts of Interest}

The authors declare that there are no conflicts of interest regarding the publication of this paper.

\section{Acknowledgments}

This work was supported by the National Natural Science Foundation of China (grant no. 61973041) and the National Key R\&D Program (grant no. 2019YFB1705403).

\section{References}

[1] T. Zan, Z.-L. Pang, M. Wang, and X.-S. Gao, "early fault diagnosis method of rolling bearings based on VMD," Journal of Beijing University of Technology, vol. 45, no. 2, pp. 103-110, 2019. 
[2] X.-Y. Zhu and Y.-J. Wang, "A method of incipient fault diagnosis of bearings based on autocorrelation analysis and MCKD," Journal of Vibration and Shock, vol. 38, no. 24, pp. 183-188, 2019.

[3] J. Ma and J. Xu, "Fault prediction algorithm for multiple mode process based on reconstruction technique," Mathematical Problems in Engineering, vol. 2015, Article ID 348729, 8 pages, 2015.

[4] R.-X. Zhuo and J.-F. Xiao, "Research on fault diagnosis method of motor bearing based on improved EEMD and SVM," Machine Building \& Automation, vol. 48, no. 1, pp. 36-39, 2019.

[5] G.-J. Tang and X.-L. Wang, "VMD and its application in early fault diagnosis of rolling bearing," Journal of Vibration Engineering, vol. 29, no. 4, pp. 638-648, 2016.

[6] S. Chen, X. Dong, Z. Peng, W. Zhang, and G. Meng, "Nonlinear chirp mode decomposition: a variational method," IEEE Transactions on Signal Processing, vol. 65, no. 22, pp. 6024-6037, 2017.

[7] Q.-Y. Lin, L.-Y. Wei, J.-K. Yae, and C.-C. Yi, "Nonlinear FM mode decomposition and application in mechanical equipment troubleshooting," Mechanical Design and Manufacturing, vol. 5, pp. 77-81, 2021.

[8] N. Mojtaba and S. S. Mahmoud, "Variational mode extraction: a new efficient method to derive respiratory signals from ECG," IEEE journal of biomedical and health informatics, vol. 22, 2017.

[9] N. Mojtaba and S. S. Mahmoud, "Successive variational mode decomposition," Signal Processing, vol. 174, 2020.

[10] R. A. Wiggins, "Minimumentropy deconvolution," Geoexploration, vol. 16, no. 1/2, pp. 21-35, 1978.

[11] M. Buzzoni, J. Antoni, and G. D’Elia, "Blind deconvolution based on cyclostationarity maximization and its application to fault identification," Journal of Sound and Vibration, vol. 432, pp. $569-601,2018$.

[12] C. A. Cabrelli, "Minimum entropy deconvolution and simplicity: a noniterative algorithm," Geophysics, vol. 50, no. 3, pp. 394-413, 1985.

[13] G. L. McDonald and Q. Zhao, "Multipoint optimal minimum entropy deconvolution and convolution fix: application to vibration fault detection," Mechanical Systems and Signal Processing, vol. 82, pp. 461-477, 2017.

[14] E. Ma, Y.-C. Li, and Z. Liu, "Extraction of rolling bearing fault characteristics based on perverted mode decomposition and Teager energy operator," Vibration and Impact, vol. 35, no. 13, pp. 134-139, 2016.

[15] D. Yan, J.-H. Yue, and J. Jia, "Extraction of rolling fault characteristics based on autocorrelation and energy operator enhancement," Vibration and Impact, vol. 40, no. 11, pp. 101-108+123, 2021.

[16] Z.-C. Qiao, Y.-Q. Liu, and Y.-Y. Liao, "Application of the improved MOMEDA method based on Teager energy operators in the troubleshooting of bearings on railway wheels," Bearings, vol. 4, pp. 43-50, 2020.

[17] H.-M. Zhang and J.-H. Zou, "Feature extraction of rolling bearing weak fault based on adaptive MCKD and ceemdan," Journal of Electronic Measurement and Instrument, vol. 33, no. 4, pp. 79-86, 2019. 